\title{
As consequências da má adesão terapêutica na artrite psoriásica no contexto da atenção primária
}

\author{
The consequences of poor therapeutic adherence in psoriatic arthritis in the context of \\ primary care
}

\section{Las consecuencias de la mala adherencia terapéutica em la artritis psoriásica em el contexto de la atención primaria}

Sílvio Antônio Gomes dos Santos Filho ${ }^{1 *}$, Lara Braga Martins ${ }^{1}$, Isabela Barandas Souza ${ }^{1}$, Fábio Alexandre Dias Reis ${ }^{1}$, Eduardo Reinaldo Silva ${ }^{1}$.

\section{RESUMO}

Objetivo: Relatar o caso de uma paciente portadora de artrite psoriática a qual evoluiu com mutilações múltiplas em mãos, articulações bilaterais de ombros, punhos bilateralmente e pés, pois não aderiu a tratamento proposto por equipe médica. Detalhamento do caso: Trata-se de um relato de caso a partir de análise de informações obtidas por meio de revisão de prontuário de uma paciente submetida a entrevistas em visita domiciliar. Tais dados colhidos são referentes ao intervalo de tempo entre 2011 e 2020, em uma cidade no interior de São Paulo. A paciente era do sexo feminino, com 62 anos, em 2020. Ela iniciou com um quadro de lesões dermatológicas descamativas em região de nádegas e couro cabeludo. Como demorou a procurar por tratamento adequado e em sequência não seguiu as orientações médicas, as lesões progrediram e a paciente começou a apresentar dor generalizada e astenia, além de sequelas tardias descritas no decorrer do trabalho. Considerações finais: Frente a dificuldade à adesão ao tratamento observada, a paciente apresentou sequelas irreversíveis em mãos e pés, como deformidades e restrição de movimentos em articulações de MMII e MMSS.

Palavras-chave: Artrite psoriática, Psoríase, Artrite, Adesão do paciente, Carência psicossocial.

\section{ABSTRACT}

Objective: Record the case of a patient with psoriatic arthritis who evolved with multiple mutilations in the hands, bilateral shoulder joints, bilateral wrists, and feet, not adhering to treatment by medical teams. Case details: This is a case report based on the analysis of information obtained by review in the medical records of a patient who was interviewed during a home visit. Such data collected are related to the time interval between 2011 and 2020, in a city in the interior of São Paulo. The patient was female, 62 years old, in 2020. She started with a picture of scaly dermatological lesions in the region of the buttocks and scalp. As it took her a long time to look for adequate treatment and in sequence she did not follow the medical guidelines, the lesions progressed and the patient began to present generalized pain and asthenia, in addition to the late sequelae described throughout the text. Final considerations: In view of the difficulty in adhering to the treatment, the patient had irreversible sequelae in her hands and feet, such as deformities and restricted movement in the lower limbs and upper limbs.

Key words: Psoriatic arthritis, Psoriasis, Arthritis, Patient compliance, Psychosocial deprivation.

\section{RESUMEN}

Objetivo: Informe el caso de un paciente con artritis psoriásica que evolucionó con múltiples mutilaciones en las manos, articulaciones bilaterales del hombre, muñecas y piezas bilaterales, y el tratamiento propuesto por el equipo médico no se cumplió. Detalles del caso: Este es un informe de caso basado en el análisis de la información obtenida a través de la revisión de los registros médicos de un paciente que fue entrevistado durante una visita domiciliaria. Dichos datos recopilados están relacionados con el intervalo de tiempo entre 2011 y 2020, en una ciudad del interior de São Paulo. La paciente era mujer, de 62 años, en 2020. Comenzó con una imagen de lesiones dermatológicas escamosas en la región de las nalgas y el cuero cabelludo. Como tardó mucho tiempo en buscar un tratamiento adecuado y en secuencia no siguió las pautas médicas, las lesiones progresaron y la paciente comenzó a presentar dolor generalizado y astenia, además de las secuelas tardías descritas durante el trabajo. Consideraciones finales: En vista de la dificultad para adherirse al tratamiento, la paciente tiene secuelas irreversibles en sus manos y pies, como deformidades y movimientos restringidos en las extremidades inferiores y superiores.

Palabras clave: Artritis psoriásica, Psoriasis, Artritis, Cooperación del paciente, Carencia psicosocial.

1Universidade de Franca (UNIFRAN), Franca - SP. *E-mail: silvioagsfilho@hotmail.com

SUBMETIDO EM: 6/2020

ACEITO EM: 7/2020

PUBLICADO EM: 7/2020 


\section{INTRODUÇÃO}

A Artrite Psoriática (APs) definida como uma artralgia de caráter inflamatório proeminente em pacientes que apresentam quadro de psoríase associado. Ela faz parte do grupo das espondiloartrites, assim como a artrite reativa, espondiloartrite associada à doença inflamatória intestinal, espondilite anquilosante e a espondiloartrite indiferenciada. No Brasil, estudos epidemiológicos constataram que a artrite psoriática é a segunda espondiloartropatia mais frequente, com prevalência de $13,7 \%$ dentre as espondiloartrites (COMISSÃO NACIONAL DE INCORPORAÇÃO DE TECNOLOGIAS NO SISTEMA ÚNICO DE SAÚDE, 2020; RUIZ DG, et al., 2012; SILVA D e PORTELINHA D, 2006; RUIZ DG, et al., 2014).

Não existe diferença de incidência quanto ao sexo. Mas sim quanto a faixa etária, os adultos são os mais acometidos. Ademais, os fatores de risco para um paciente com psoríase apresentar artrite são: envolvimento cutâneo mais extenso, história familiar de artrite psoriática e presença de lesões em unhas. Em relação à transmissão genética, o risco entre irmãos de ter a doença é superior a $27 \%$, comparado com o risco da população geral. Além disso, o risco de parentes de primeiro grau de indivíduos acometidos por tal patologia de virem a desenvolvê-la é de 19 vezes a mais (COMISSÃO NACIONAL DE INCORPORAÇÃO DE TECNOLOGIAS NO SISTEMA ÚNICO DE SAÚDE, 2020; RUIZ DG, et al., 2014).

Existem algumas formas de manifestações clínicas dessa artrite. Assim, podemos classificá-la em oligoarticular (mais comum) que envolve menos de cinco articulações, sendo as mais atingidas tornozelos e joelhos. Também, em poliarticular, que se assemelha a artrite reumatoide, podendo evoluir para deformidades após longo prazo. Por fim, a forma mutilante, a qual o paciente apresenta grandes destruições articulares, principalmente em pés e mãos. Outrossim, é possível apresentar uma forma mais rara, envolvendo a coluna vertebral (SOCIEDADE BRASILEIRA DE REUMATOLOGIA, 2020; GLADMAN DD, et al., 2005; KRAKOWSKI $P$, et al., 2019). Outras características apresentadas por tais indivíduos são entesites, tenossinovites e dactilites. Do mesmo modo, podem desenvolver manifestações extra-articulares típicas, incluindo o envolvimento ungueal, ocular, cutâneo, cardiovascular, renal e pulmonar (COMISSÃO NACIONAL DE INCORPORAÇÃO DE TECNOLOGIAS NO SISTEMA ÚNICO DE SAÚDE, 2020; GLADMAN DD, et al., 2005).

Para a obtenção do diagnóstico, leva-se em consideração principalmente as evidências clínicas. Sendo que, as características mais importantes são o acometimento de articulações dos ombros, membros inferiores e pequenas articulações das mãos, fator reumatóide negativo, além da presença de entesites e tendinites. Alguns exames podem se mostrar alterados, como os substratos de atividade inflamatória (velocidade de hemossedimentação e a proteína $C$ reativa), a glicemia (o risco de diabetes mellitus é maior nesses pacientes) e a dosagem de ácido úrico. Em casos duvidosos, é possível biopsiar a lesão articular, a fim de realizar exame histopatológico, visando uma análise mais detalhada (SOCIEDADE BRASILEIRA DE REUMATOLOGIA, 2020).

Em vista da escolha terapêutica, deve-se avaliar a presença de entesites, dactilites, lesões nas unhas, acometimento articular periférico e axial. Assim, pode ser feito o tratamento convencional, com o uso de antiinflamatórios não esteroidais (AINES) e medicações denominadas modificadores do curso da doença. Já em casos mais graves, pode ser necessário o uso de agentes biológicos, incluindo etanercepte, golimumabe, infliximabe, dentre outros. Os pacientes os quais desenvolvem deformidades nos pés e/ou mãos podem se beneficiar de cirurgias, especialmente aquelas que visam a colocação de prótese, como no quadril ou joelho (SOCIEDADE BRASILEIRA DE REUMATOLOGIA, 2020).

Diante tais informações, o objetivo deste estudo foi avaliar um caso de artrite psoriática em uma paciente que apresentou um quadro clássico da doença e não procurou o devido tratamento para tratar as lesões. A justificativa para tal trabalho baseia-se na importância de levar informações para a população acadêmica e médica sobre a adesão ao tratamento correto, a fim de evitar possíveis deformidades irreversíveis.

\section{DETALHAMENTO DO CASO}

As informações contidas neste trabalho foram obtidas por meio de revisão do prontuário da paciente no qual havia dados de visitas domiciliares. Assim como, registro fotográfico de exames complementares e de lesões (fotografias autorizadas pela paciente). Também, há relato de informações obtidas por meio de revisão 
de literatura. Ademais, este trabalho foi aprovado pelo Comitê de Ética em Pesquisa, protocolo CAAE N ${ }^{\circ}$ 30608320.7.0000.5495 e obedeceu aos princípios éticos e legais da resolução no 466/12.

A paciente em análise é do sexo feminino, em 2020 com 62 anos, procedente do interior de São Paulo. Ela iniciou quadro de lesões dermatológicas descamativas em região de nádegas e couro cabeludo há 12 anos, porém não procurou atendimento para tratar tais lesões. Após dois anos sem tratamento, o quadro evoluiu com dor generalizada, astenia e remissão das lesões dermatológicas. A dor apresentava piora progressiva, o que levava a restrição de movimento e dificuldade para realizar as atividades básicas da vida diária.

Em março de 2010, procurou atendimento médico com queixa de dor articular importante que aliviava com uso de AINES. Após o primeiro atendimento, outras inúmeras consultas em decorrência de dor generalizada, lombalgia, dorsalgia, dificuldade de movimentação e restrição das atividades vieram a ocorrer. No ano de 2011, em nova consulta, foi evidenciada perda ponderal de $12 \mathrm{~kg}$ em 6 meses, também queixou de poliartralgia. Por isso, foi receitado prednisona, cloroquina, indometacina e ranitidina.

Em junho de 2012, foram solicitados alguns exames e por meio dos resultados obtidos, foi possível verificar um aumento da uréia e da alanina aminotransferase, de acordo com os valores de referência do Ministério da Saúde. Os resultados estão demonstrados na Tabela 1.

Tabela 1 - Exames laboratoriais realizados e os respectivos valores de referência.

\begin{tabular}{ccc}
\hline Exame & Valor encontrado & Valores de referência \\
\hline Ácido úrico & 5,7 & $2,5-8,0$ \\
Ureia & 33,4 & $8,0-20,0$ \\
Creatinina & 1,19 & $0,7-1,3$ \\
Aspartato aminotransferase & 33,4 & $0-35$ \\
Alanina aminotransferase & 38 & $0-35$ \\
\hline
\end{tabular}

Legenda: Valores de referências baseados em: MANUAL MSD, 2020.

Fonte: Filho SAGS, et al., 2020.

Além disso, realizou radiografia de membros superiores e inferiores. Nas mãos, apresentou redução da mineralização óssea, redução do espaço articular intercarpal e entre o rádio e o carpo, absorção de extremidade ulnar e deformidades dos dedos. Em ambos os pés houve uma redução da mineralização óssea, hálux valgo e redução do espaço articular interfalangiano, além de desvio medial das cabeças dos metatarsos (Figuras 1A e B).

Figura 1 - Imagens representativas das radiografias realizadas. A - Radiografia dos pés; B - Radiografia das mãos e punhos.
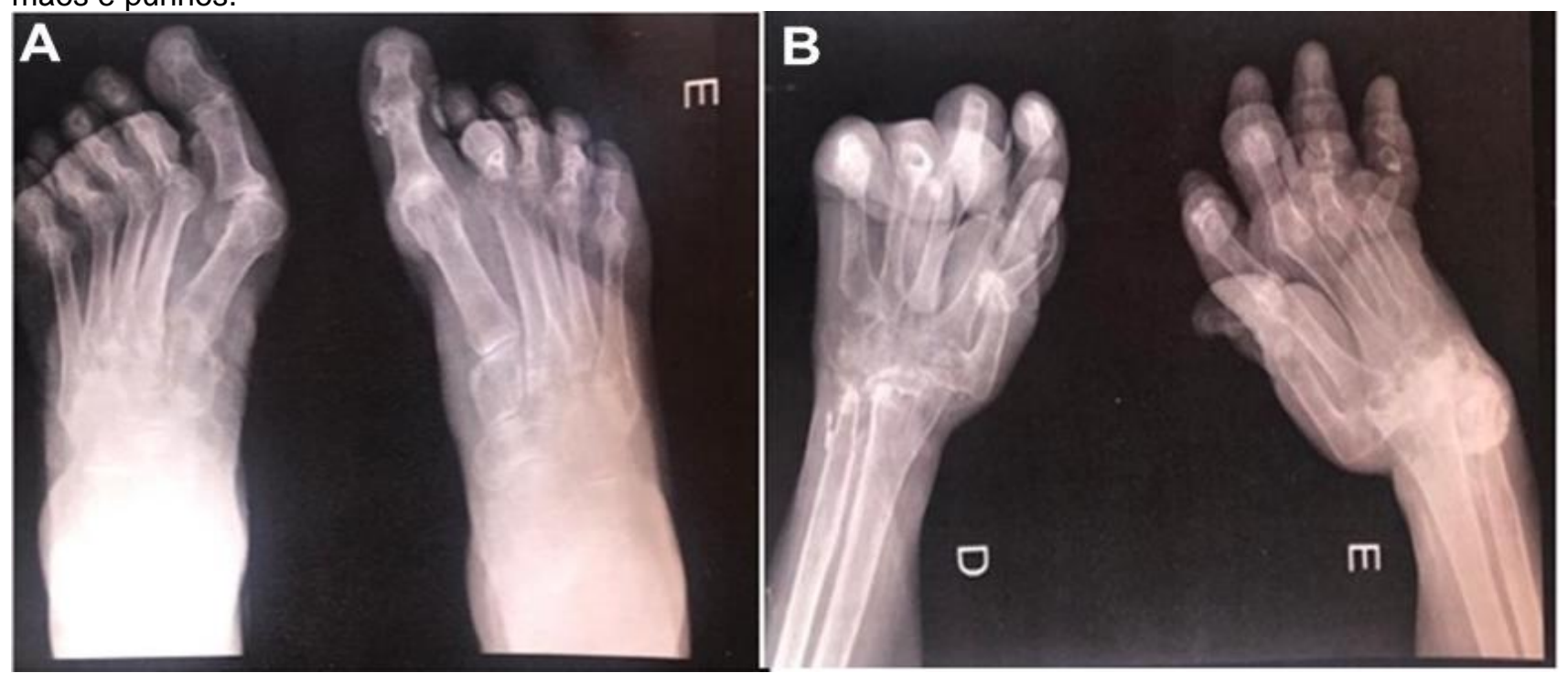

Legenda: $\mathrm{D}=$ Direito; $\mathrm{E}=$ Esquerdo.

Fonte: Filho SAGS, et al., 2020. 
No mês de maio de 2013, em visita domiciliar a paciente apresentava em membro inferior esquerdo (MIE) uma lesão com tecido de granulação, exsudativa e sem odor fétido. Devido a isso, foi utilizado soro fisiológico $0,9 \%$ morno, associado à colagenase pomada em curativo. Assim como, orientado os familiares para cuidados gerais diários da ferida.

Em novembro de 2013, começou a apresentar deformidades e atrofias em mãos e pés (Figuras 2A, B, C e D), acentuando a dor. Porém, com exercícios de fisioterapia, houve melhora parcial das atrofias. No exame físico, foi registrado algumas restrições de movimento em ombros e movimentação diminuída de cotovelo, joelho e tornozelo, além de um leve edema de membros inferiores (MMII). Foi orientada a realizar alongamentos diários, evitar permanecer no leito por longos períodos, manter o tratamento fisioterapêutico semanal e manter MMII elevados quando apresentar edema.

Figura 2 - Imagens das deformidades e atrofias. A e B: Deformidade em articulação de punhos. C e D: Deformidade em quirodáctilos.
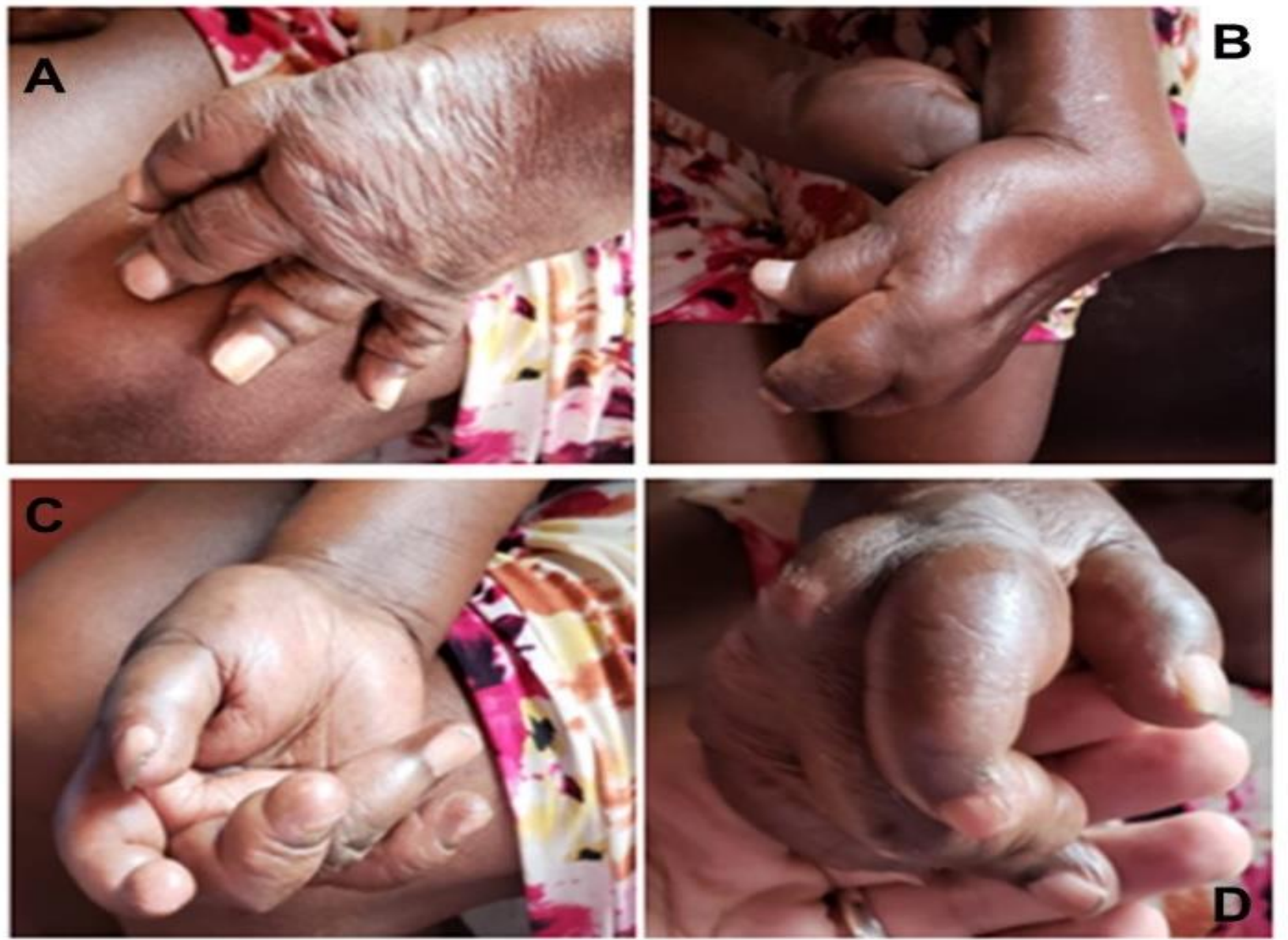

Fonte: Filho SAGS, et al., 2020.

Em fevereiro de 2014, foi diagnosticada com artrite reumatoide. Por isso, prescrito AINES, corticosteroides, antidepressivo tricíclico e antagonista de Receptor H2 (para proteção da mucosa gástrica dos efeitos adversos). Em março de 2019, paciente retorna em consulta para acompanhamento de sequela de poliartralgia. Nesta ocasião, foi prescrito imipramina para controle da dor. Bem como, encaminhada para seguimento com reumatologista. Após oito meses, comparece a outra consulta, apresentando as sequelas de artrite psoriática e dor crônica.

Por fim, em última visita domiciliar realizada no dia 30 de janeiro de 2020, a paciente evoluiu com quadro de desalinhamento da anatomia dos quirodáctilos, edema de MMSS (membros superiores) e sensação de dor do tipo queimação em mãos. Havia também lesões psoriáticas descamativas e pruriginosas em couro cabeludo, região anal e perianal, sem prurido. Em virtude disso, foi prescrito metotrexato (Figura 3 A e B). 
Figura 3 - Presença de lesões descamativas. A - Lesões descamativas em nádegas. B - Lesões descamativas em couro cabeludo.
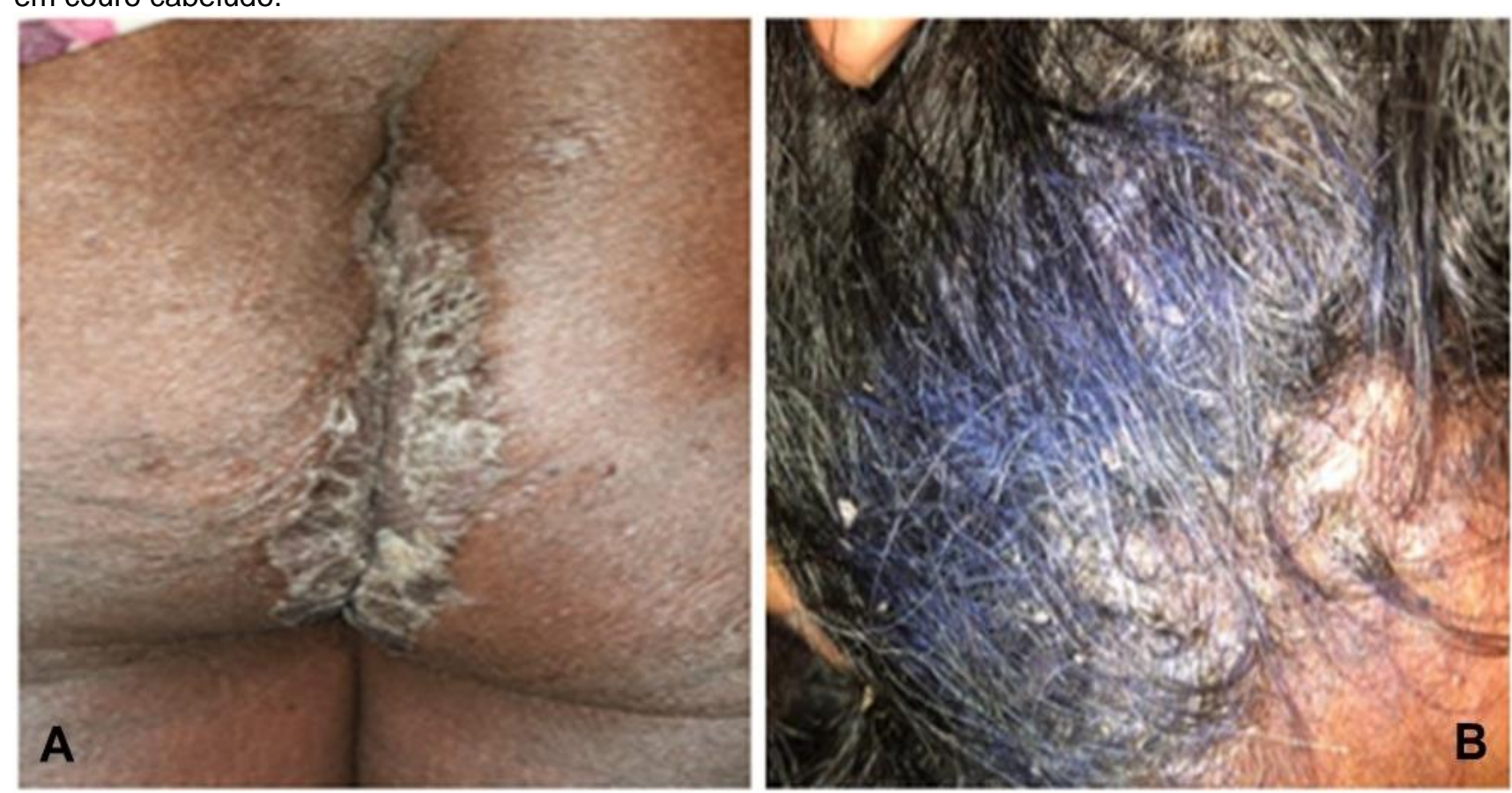

Fonte: Filho SAGS, et al., 2020.

Nesta consulta, ao exame osteomuscular, apresentou mutilações múltiplas e deformidade em mãos e pés bilateralmente. Presença de restrição de movimentação passiva e ativa em articulações de MMII e membros superiores (MMSS), assim como presença de dor a movimentação.

\section{DISCUSSÃO}

A APs é uma doença articular inflamatória, comumente poliarticular que se manifesta por sinais flogísticos gerais e dor. É associada a psoríase, caracterizada por aparecimento na pele de placas eritematosas e descamativas, apresentando um contorno bem delimitado. Estas lesões se localizam com maior frequência no couro cabeludo, cotovelos e joelhos, mas podem surgir em qualquer parte do corpo. A etiologia ainda não é conhecida, porém sabe-se que existem fatores genéticos, imunológicos e ambientais que contribuem para o aparecimento desta doença, sendo aventada a possibilidade de predisposição familiar. (COMISSÃO NACIONAL DE INCORPORAÇÃO DE TECNOLOGIAS NO SISTEMA ÚNICO DE SAÚDE, 2020; SILVA D e PORTELINHA D, 2006; RUIZ DG, et al., 2012; SCHAINBERG CG, 2012).

Há ainda associação da APs com alguns antígenos de histocompatibilidade, sendo a presença dos antígenos HLA-B27 (human leukocyte antigen subtipo B27) e subtipo B39 vinculado à gravidade e à evolução da doença. Apesar disso, a APs não é considerada uma doença autoimune, apesar de apresentar em sua fisiopatologia aumento de citocinas inflamatórias, como o fator de necrose tumoral $\alpha$ (TNFa) e de interleucinas pró-inflamatórias (principalmente das IL-1, IL-6, IL-8) nas articulações acometidas (RUIZ DG, et al., 2012; SILVA D e PORTELINHA D, 2006; SCHAINBERG CG, 2012).

Existem também fatores ambientais que podem estar envolvidos, sendo eles microorganismos (bactérias e vírus), traumas orgânicos e o estresse psicológico. Todavia, a gênese e a influência desses fatores permanecem desconhecidas (SILVA D e PORTELINHA D, 2006).

Quanto à psoríase, seus subtipos são divididos em psoríase vulgar, psoríase guttata, psoríase pustulosa, psoríase invertida, psoríase eritrodérmica, e por fim, a psoríase artropática (ou artrite psoriática) abordada neste relato, a qual se manifesta com reação inflamatória cutânea associada à descamação e a fortes dores articulares, principalmente nas articulações do quadril, coluna, mãos e pés. O acometimento articular pode ser gradual e levar a rigidez de movimento progressivo e dificuldade funcional. Tais lesões psoriáticas têm 
como perfil a capacidade de se tornarem crônicas, assim como de serem recorrentes (RUIZ DG, et al., 2012; SCHAINBERG CG, 2012; SILVA D e PORTELINHA D, 2006; SOCIEDADE BRASILEIRA DE DERMATOLOGIA, 2020; ARMELIN E, 2016).

Ainda a respeito da artrite psoriática, ela também pode ser distal, atingindo falanges distais das mãos, pés e leitos ungueais. Assim como, pode apresentar acometimento da coluna vertebral, principalmente na transição toracolombar. Também, em seu estado mais grave, a artrite psoriática pode se manifestar com aspecto mutilante, apesar de ser menos comum. Esse tipo mais intenso pode envolver as articulações das mãos e pés, mas pode atingir articulações diversas progressivamente tendo como resultado deformações irreversíveis e dificuldades funcionais para o indivíduo (SOCIEDADE BRASILEIRA DE REUMATOLOGIA, 2020).

O quadro da paciente relatada não foge das manifestações expostas na literatura. De início, a mesma apresentou as típicas lesões dermatológicas descamativas em região de nádegas e couro cabeludo. Em sequência, evoluiu para dor articular generalizada, atrofias em mãos e pés, associado a restrição gradual de movimentação. Tardiamente, houve piora do quadro, pois também passou a apresentar alteração no alinhamento da anatomia dos quirodáctilos. Consequentemente, pode-se inferir que se trata de uma artrite psoriática de aspecto mutilante.

Existem diversos critérios de diagnóstico para APs, contudo há evidências literárias que o estudo multicêntrico Classification Criteria for Psoriatic Arthritis (CASPAR) realizado em 2006 apresentou vantagens em relação aos demais, permitindo diagnóstico precoce, mesmo com lesões dermatológicas pequenas. Nessa classificação, o paciente deve conter obrigatoriamente um quadro de artrite prévia e atingir pelo menos três pontos nos demais critérios avaliados: Psoríase atual (2 pontos), história prévia de psoríase (1 ponto), história familiar de psoríase ( 1 ponto), distrofia ungueal ( 1 ponto), FR negativo ( 1 ponto), dactilite e/ ou lesões radiológicas típicas em mãos e pés (1 ponto). No caso da paciente deste relato, a pontuação final seria de cinco pontos (RUIZ DG, et al., 2012; SCHAINBERG CG, 2012; MINISTÉRIO DA SAÚDE, 2018; TAYLOR W, et al., 2006).

Fazendo uma comparação entre as lesões radiológicas típicas da literatura e as lesões da paciente do caso, notamos uma grande similaridade. Em ambos, a falange distal está consumida, existe também dissolução principalmente dos metatarsos e a mão ocupa um aspecto arredondado, equivalente ao que é denominado "óculo de ópera", característica comum em casos de artrite psoriática mutilante (GLADMAN DD, et al., 2005; KRAKOWSKI P, et al., 2019; MOURA S e PEREIRA C, 2015).

O tratamento da APs tem como objetivo aliviar a dor, prevenir erosões ósseas e evitar incapacidade de mobilização. Pode ser não-medicamentoso e medicamentoso, normalmente associados para buscar um melhor resultado e adequação terapêutica (SOCIEDADE BRASILEIRA DE REUMATOLOGIA, 2020; SILVA D e PORTELINHA D, 2006; KRAKOWSKI P, et al., 2019).

O tratamento não-medicamentoso engloba a fisioterapia individualizada, a fim de atender às necessidades particulares de cada paciente. Durante a fase aguda, é orientado repouso relativo com fisioterapia passiva visando prevenir atrofias musculares ou rigidez das articulações envolvidas (SOCIEDADE BRASILEIRA DE REUMATOLOGIA, 2020; SILVA D e PORTELINHA D, 2006).

O Tratamento Farmacológico visa o alívio da dor. Para isso, são utilizados AINES e Bloqueadores de TNF, depletores de linfócito $B$ e moduladores de co-estimulação. Dentro da classe dos Bloqueadores de TNF está o Adalimumabe, Etanercepte, Golimumabe e Infliximabe. Todos com exceção ao Infliximabe, podem ser associados ao metotrexato. Já, os depletores de linfócito B são representados pelo Rituximabe. Por fim, o representado dos moduladores de co-estimulação é o Abatacepte terapêutica (SOCIEDADE BRASILEIRA DE REUMATOLOGIA, 2020; SILVA D e PORTELINHA D, 2006; PLANSERV, 2012; CARNEIRO S, et al., 2013).

Procedimentos cirúrgicos também podem ser utilizados. Todavia, devem ser restritos aos casos de limitação importante da funcionalidade articular, ou para substituição de próteses já inseridas em articulações, sendo as coxofemorais as mais sujeitas a esse tipo de tratamento (SOCIEDADE BRASILEIRA DE REUMATOLOGIA, 2020; SILVA D e PORTELINHA D, 2006; KRAKOWSKI P, et al., 2019). 
No caso da paciente, foram realizadas sessões de fisioterapia e orientado evitar permanecer no leito por longos períodos. Também, para manter MMII elevados quando apresentar edema. Além disso, foi feito o uso de AINES para melhora da dor (indometacina), corticosteróide (prednisona), antidepressivo tricíclico (imipramina), antagonista de Receptor $\mathrm{H} 2$ (ranitidina) e metotrexato. Medicamentos como os bloqueadores de TNF, depletores de linfócito $\mathrm{B}$, ou moduladores de co-estimulação não foram prescritos, mas poderiam ter trazido grandes benefícios para o tratamento. Ademais, poderiam ser realizados procedimentos cirúrgicos em decorrência de suas restrições de movimentos, ou então, fazer a utilização de próteses.

Após a revisão de literatura realizada neste trabalho, foi possível concluir a importância da adesão correta ao tratamento para pacientes com artrite psoriásica. A fim de, evitar sequelas tardias irreversíveis, como deformidades e restrições de movimentos em articulações, como as que ocorreram com a paciente deste relato. Além disso, foi visto que a visita domiciliar tem o objetivo de promover a aproximação dos profissionais ao contexto individual, promovendo a valorização do contexto inserido e propondo o melhor tratamento para o paciente envolvido.

\section{REFERÊNCIAS}

1. COMISSÃO NACIONAL DE INCORPORAÇÃO DE TECNOLOGIAS NO SISTEMA ÚNICO DE SAÚDE. In: Protocolo clínico e diretrizes terapêuticas: artrite psoríaca. Brasil. Disponível em: http://conitec.gov.br/images/Protocolos/Artrite-Psoriaca.pdf. Acesso em 29 maio. 2020.

2. RUIZ DG, et al. Artrite psoriásica: entidade clínica distinta da psoríase?. Rev Bras Reumatol, 2012;52(4):623-638.

3. SOCIEDADE BRASILEIRA DE REUMATOLOGIA. In: Artrite psoriásica: manejo e tratamento. Brasil. Disponível em: https://diretrizes.amb.org.br/_DIRETRIZES/artrite_psoriasica_manejo_e_tratamento/files/assets/common/download s/publication.pdf. Acesso em 29 maio.2020.

4. SILVA D, PORTELINHA D. Artrite Psoriática. Nedai, 2006.

5. RUIZ DG, et al. Caracterização clínica de pacientes com artrite psoriásica. Rev Soc Bras Clin Med, 2014; 12(2).

6. SCHAINBERG CG. Conceitos atuais e relevantes sobre artrite psoriásica. Rev Bras Reumatol, 2012; 52(1):92-106.

7. MANUAL MDS. In: Exames de sangue: valores normais. Brasil. Disponível em: https://www.msdmanuals.com/pt$\mathrm{pt} /$ profissional/ap\%C3\%AAndices/valores-laboratoriais-normais/exames-de-sangue-valores-normais. Acesso em 12 de julho. 2020.

8. MOURA S, PEREIRA C. Artrite Mutilante. Galicia Clin, 2015; 76(4): 183.

9. PLANSERV. Protocolo clínico e diretrizes terapêuticas para tratamento da artrite psoriásica. 2nd ed. Salvador: SAEB/CGPS,2012.

10. SOCIEDADE BRASILEIRA DE REUMATOLOGIA. In: Artrite psoriásica. Brasil. Disponível em: https://www.reumatologia.org.br/download/artrite-psoriasica-a4/. Acesso em 29 maio. 2020.

11. SOCIEDADE BRASILEIRA DE DERMATOLOGIA. In: Psoríase. Brasil. Disponível em: https://www. sbd.org.br/dermatologia/pele/doencas-e-problemas/psoriase/18/. Acesso em 29 maio. 2020.

12. CARNEIRO S, et al. Recomendações sobre diagnóstico e tratamento da artrite psoriásica. Rev Bras Reumatol, 2013; 53(3):227-241.

13. MINISTÉRIO DA SAÚDE. Portaria conjunta no 26, de24deoutubrode2018. Brasil. Aprova o protocolo clínico e diretrizes terapêuticas da artrite psoríaca. Diário Oficial União, 2018.

14. ARMELIN E. Psoríase e suas principais características. Dissertação (Bacharel em Enfermagem). Instituto de Ensino Superior de Londrina, Londrina, 2016.

15. GLADMAN DD, et al. Psoriatic arthritis: epidemiology, clinical features, course, and outcome. Ann RheumDis, 2005; 64(Suppl II):ii14-ii1.

16. TAYLOR W, et al. Classification Criteria for Psoriatic Arthritis. Arthritis\&Rheumatism, 2006; 54(8): 2665-2673.

17. KRAKOWSKI P, et al. Psoriaticarthritis - new perspectives. Arch Med Sci, 2019; 15(3): 580-589. 